\title{
AN EXTENSION OF THE NOMOGRAMIC INSTRUMENT
}

HARRY D. RUDERMAN

The usual nomogram involves three variables and has the straight line for its instrument. We may say, therefore, that its instrument is a member of the family of straight lines

$$
A_{1} x_{1}+A_{2} x_{2}+A_{3}=0 .
$$

More generally, a hyperplane in $n$-dimensional space is usually necessary for $n+1$ variables. By permitting more complicated instruments it may be possible to reduce the number of dimensions in which the instrument operates. The theorem to be given does precisely this by selecting the instrument from a certain family of hypersurfaces.

Definition. If $f$ is a function of $n$ variables $s_{1}, \cdots, s_{n}$ and can be expressed by an $n$ by $n$ determinant $\left(V_{i j}\right)$ where $V_{i j}$ is a function of $s_{i}$ only, then $f$ will be called a nomogramic disjunctive function, or simply an $\mathrm{N}$-function.

Definition. Let $R_{i}^{k}$ be a function of the single variable $s_{i}$ for $k=1, \cdots, m$ and for $i=1, \cdots, n$. Let $g_{j}$ be a function of $m$ variables $u_{1}, \cdots, u_{m}$ for $j=1, \cdots, n$. We shall say that the triple $\left(m, R_{i}^{\mathbf{k}}, g_{j}\right)$ belongs to $f$ if $f$ is an $\mathrm{N}$-function with

$$
V_{i j}=g_{i}\left(R_{i}^{1}, \cdots, R_{i}^{m}\right) \quad \text { for } i, j=1, \cdots, n .
$$

( $k$ is a superscript.)

Lemma. Every N-function has at least one triple.

Proof. Let $f$ be an $\mathrm{N}$-function. Then take

$$
m=n, \quad g_{i}\left(u_{1}, \cdots, u_{n}\right)=u_{j}, \quad R_{i}^{k}=V_{i k} .
$$

Theorem. Let $\left(m, R_{i}^{\mathbf{k}}, g_{j}\right)$ belong to $f$. Let $f$ be defined on $D$, a subset of $n$-dimensional euclidean space $E^{n}$. Let $T_{1}, \cdots, T_{m}$ be $m$ arbitrary functions of a single variable which have inverses on the domains considered. Let $C_{1}, \cdots, C_{n}$ be $n$ curves in $E^{m}$ given parametrically by

$$
C_{i}: \quad x_{k}=T_{k}^{-1}\left(R_{i}^{k}\left(s_{i}\right)\right), \quad k=1, \cdots, m ; i=1, \cdots, n .
$$

Finally, let $F$ be the family of hypersurfaces indicated by

$$
A_{1} g_{1}\left(T_{1}\left(x_{1}\right), \cdots, T_{m}\left(x_{m}\right)\right)+\cdots+A_{n} g_{n}\left(T_{1}\left(x_{1}\right), \cdots, T_{m}\left(x_{m}\right)\right)=0
$$

where the A's are in a field embedding the ranges of the $g$ 's. Then for

Received by the editors February 14, 1950. 
each $\left(s_{1}, \cdots, s_{n}\right)$ which annuls $f$ there is a set of $n$ points $\left(x_{1}, \cdots, x_{m}\right)_{i}$ on $C_{i}$ for $i=1, \cdots, n$ which lie on a single member of $F$. The coordinates of these $n$ points are obtained from the parametric expressions for the curves.

Conversely, if a member of $F$ intersects $C_{1}, \cdots, C_{n}$ in the $n$ points $\left(x_{1}, \cdots, x_{m}\right)_{i}$ for $i=1, \cdots, n$, respectively, which arise from $s_{1}, \cdots, s_{n}$, respectively, in constructing the curves, then $f$ is annulled by $\left(s_{1}, \cdots, s_{n}\right)$.

Proof. Let $f(\bar{s})=0$ where $\bar{s}=\left(\bar{s}_{1}, \cdots, \bar{s}_{n}\right)$. Then the determinant expression for $f$ vanishes for $\bar{s}$. Each $\bar{s}_{i}$ determines a single point on $C_{i}$ namely, $\left(\bar{x}_{1}, \cdots, \bar{x}_{m}\right)_{i}$. Put these $n$ values one at a time into the general expression for $F$ and consider $A_{1}, \cdots, A_{n}$ as the unknowns to be solved for. Then the resulting set of $n$ equations has a coefficient determinant which vanishes as it is precisely $f(\bar{s})$. Hence, there exists a nontrivial set of values for $A_{1}, \cdots, A_{n}$ which determines a member of $F$, and this member vanishes on all $n$ points $\left(\bar{x}_{1}, \cdots, \bar{x}_{m}\right)_{i}$, $i=1, \cdots, n$.

For the converse, let $h$, a member of $F$, intersect $C_{i}$ in $\left(\bar{x}_{1}, \cdots, \bar{x}_{m}\right)_{i}$ which gives rise to $\bar{s}_{i}, i=1, \cdots, n$. Let the point $\left(\bar{s}_{1}, \cdots, \bar{s}_{n}\right)$ belong to $D$. Now each $\left(\bar{x}_{1}, \cdots, \bar{x}_{m}\right)_{i}$ annuls $h$. Consider $A_{1}, \cdots, A_{n}$ as unknowns in these $n$ vanishing expressions, then we have a solution to a set of $n$ homogeneous linear equations and this solution will not be trivial if we avoid the zero member of $F$. Hence, the determinant of the coefficients of the $A$ 's, which is precisely $f(\bar{s})$, must vanish. This completes the proof of the theorem.

\section{REFERENCES}

1. D. F. Barrow, Expressing a function of three variables in nomograph form, Duke Math. J. vol. 15 (1948) pp. 433-437.

2. J. M. Thomas, Nomographic disjunction, Duke Math. J. vol. 16 (1949) pp. 419-432.

3. M. D'Ocagne, Traité de nomographie, $2 \mathrm{~d}$ ed., Paris, 1921.

New York City. 